\title{
El papel de la información económica como generador de conocimiento en el proceso de predicción: Comparaciones empíricas del crecimiento del PIB regional $^{*}$
}

\author{
ANA M. LÓPEZ \\ Universidad Autónoma de Madrid, Instituto L.R. Klein, C/ Francisco Tomas y Valiente,5, 28049 \\ Madrid,España.E-mail: ana.lopez@uam.es
}

\begin{abstract}
RESUMEN
La era actual y el desarrollo de internet han propiciado la accesibilidad a diferentes fuentes de información estadística. Disponemos de más datos cuantitativos, más información, aunque no siempre significan más conocimiento. El gran reto es convertir los datos disponibles en información y ésta en conocimiento. Una parcela interesante del conocimiento en economía es la que nos proporciona la posibilidad de ahondar en el ámbito de la predicción, favorecida por la disponibilidad de datos y de programas informáticos específicos. La principal magnitud que aún se sigue empleando para calificar la prosperidad económica de un país o área territorial es el Producto Interior Bruto (PIB). Su tasa de variación precisa ser interpretada correctamente para extraer conclusiones relevantes en la toma de decisiones económicas, en la generación y difusión de conocimiento. Revisamos los principales aspectos a considerar en el análisis de predicciones del PIB regional en España, con la finalidad de contribuir a esclarecer el interés por la combinación de predicciones alternativas o la apuesta por una predicción en particular por su mayor fiabilidad.
\end{abstract}

Palabras clave: PIB, actividad económica, predicción económica, modelización regional.

\section{The Role of Economic Information as a Generator of Knowledge in the Forecasting Process: Empirical Comparisons of Spanish Regional GDP Growth}

\begin{abstract}
The current age and the internet development give raise accessibility to different sources of statistical information. We have more information, though not always it means an increase of knowledge. The great challenge is to turn the available economic data into information and this one into economic knowledge. The possibility of going more deeply into the area of economic forecasting is an interesting focus of knowledge in economic science, supported by the availability of information and specific software. The gross domestic product (GDP) still continues being used as the main magnitude to qualify the economic prosperity of a country or geographic area. It is necessary to interpret correctly the GDP variation in order to extract relevant conclusions for the economic decisions, for the generation and dissemination of knowledge. We review some relevant aspects to consider in the process of regional economic forecasting: forecast combination or to choose the most accurate prediction.
\end{abstract}

Keywords: GDP, economic activity, regional forecasting, regional modelling.

Clasificación JEL: C53

\footnotetext{
* La autora agradece los comentarios y sugerencias de dos evaluadores anónimos que han contribuido a la mejora de la versión final del trabajo que aquí se presenta.
}

Artículo recibido en mayo de 2016 y aceptado en julio de 2016

Artículo disponible en versión electrónica en la página www.revista-eea.net, ref. ə-34304 


\section{SOBRE DATOS, INFORMACIÓN Y CONOCIMIENTO EN ECONOMÍA}

Con cierta frecuencia, en el lenguaje cotidiano se utilizan de forma indistinta los términos datos, información y conocimiento como si se tratasen de sinónimos o descripciones de una misma realidad. Sin embargo, la precisión del lenguaje determina la diferencia entre los tres conceptos señalados. En su definición más elemental, los datos son simplemente valores numéricos que representan una realidad abstracta.

Como exponen Davenport y Prusak (1998) "el conocimiento no es datos ni información, a pesar de que está relacionado con ambos, y las diferencias entre estos términos son, a menudo, una cuestión de nivel”. Los autores diferencian los tres términos, matizando que dato es "un conjunto de hechos distintos y objetivos, relativos a eventos”. Los datos no tienen significado en sí mismos, describen una parte de la realidad y no proporcionan interpretación adicional. La interpretación de un dato aislado no tiene una especial relevancia o no permite explicar suficientemente una situación concreta.

Aunque los datos pueden ser considerados como la base de la creación de información, lo cierto es que se convierten en información cuando su productor les añade un significado. La toma de decisiones se basa en los datos, en los juicios de valor o diagnóstico que pueda establecerse al analizarlos, en la interpretación que se hace de ellos. La información se caracteriza por la existencia de un mensaje a transmitir a un receptor por parte de un emisor. La información tiene, por tanto, significado en términos de relevancia y propósito. El mensaje a transmitir tiene una determinada organización. Siguiendo a Davenport y Prusak “... los datos se convierten en información cuando su creador añade significado. Transformamos los datos en información mediante la adición de valor de diversas maneras: conceptualizando, categorizando, calculando, corrigiendo y condensando". Se trata de identificar el propósito para el que los datos fueron generados, que se corresponden con unas determinadas unidades de análisis. Los datos pueden analizarse desde la óptica matemática o estadística, y su revisión conduce a la corrección de posibles errores iniciales de cómputo.

El tercer nivel, el del conocimiento, es más profundo y amplio que los datos y la información. Para Davenport y Prusak el conocimiento se origina en la mente de los conocedores, "es una mezcla de experiencia, valores, información y saber hacer que sirve como marco para la incorporación de nuevas experiencias e información, y es útil para la acción”. Se establece, por tanto, una cierta jerarquía donde los datos objetivos son la fuente del conocimiento.

La era actual y el desarrollo de internet han favorecido y aumentado la accesibilidad a diferentes fuentes de información estadística, tanto de ámbito nacional como internacional. El uso de las nuevas tecnologías de información y 
comunicación, las TIC, posibilita una mayor accesibilidad a los datos, a su utilización, a la mejora en la gestión de los datos, pero no implica necesariamente que, por ello, se mejore el nivel de información. Disponemos de más datos, más información aunque no siempre significan más conocimiento, al menos de forma directa, pues hay que saber cómo procesarla. El mero acceso a los datos y a la información no garantiza que se genere conocimiento o se mejore el ya existente.

En el ámbito particular de la economía, la sucesión entre los tres conceptos definidos es prácticamente la misma, donde cada nivel es construido sobre la base del anterior, dotando al conocimiento de la máxima importancia en todo el proceso. Ya en los trabajos clásicos de Marshall (1890), reconocido por su aportación a la concepción de la actual "economía del conocimiento", sostenía que el conocimiento es el factor de producción más poderoso al identificarlo como cuarto factor: "El conocimiento es nuestra máquina de producción más potente; nos permite someter a la naturaleza y obligarla a satisfacer nuestras necesidades”. De igual modo, Hayek ${ }^{1}$ (1945) explicaba que uno de los principales problemas de la política económica se debía a la necesidad de determinar la mejor forma de utilizar un conocimiento disperso entre toda la población. Manifestaba que "el complejo de decisiones interrelacionadas relativas a la asignación de los recursos disponibles en la economía debe estar basado en el conocimiento".

Figura 1

El proceso de generación de conocimiento

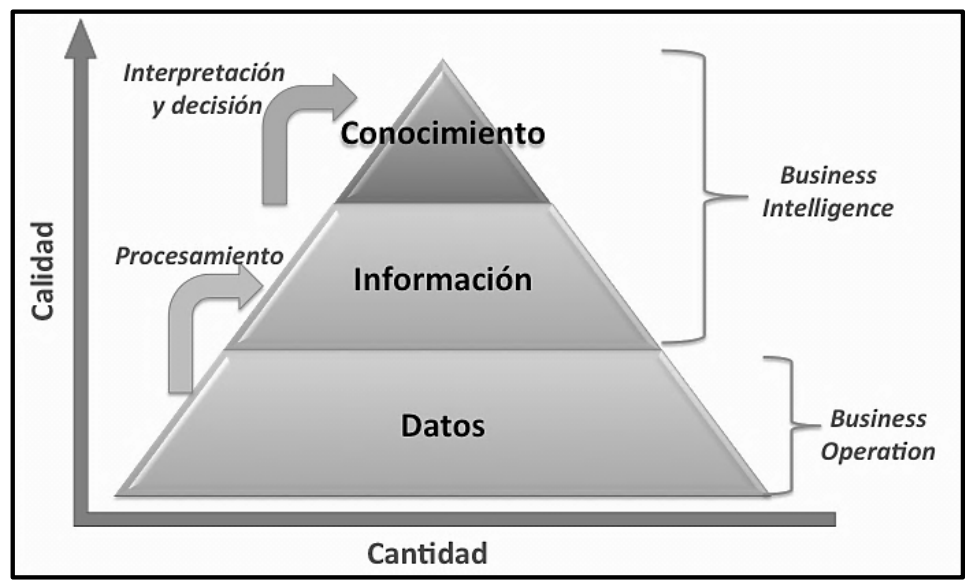

Fuente: Elaboración propia a partir de Davenport y Prusak.

${ }^{1}$ Friedrich August von Hayek obtuvo el Premio Nobel de Economía en 1974, compartido con Gunnar Myrdal, por "su trabajo pionero en la teoría del dinero y las fluctuaciones económicas y sus análisis de la interdependencia de los fenómenos económicos, sociales e institucionales”. 
En el contexto y terminología actual, el enfoque citado de Davenport y Prusak se asocia con las áreas de business operation y business intelligence (Figura 1). Por tanto, el gran reto es convertir los datos (cantidad) en información y ésta, a su vez en conocimiento (calidad).

En economía, la elaboración de indicadores compuestos y modelos (información) ayudan en el proceso de interpretación precedente a la generación del conocimiento. Un paso previo a la experiencia de modelización de la economía y posterior elaboración de predicciones, es la obtención de los datos, a los que nos referimos como la información estadística precisa para el buen desempeño de esta acción. La producción de información puede clasificarse como primaria o derivada. La publicación de esta información estadística hoy día es tanto física, en el tradicional soporte papel o digital, o con medios de acceso electrónicos, disponible en modo on- line a través de internet.

La difusión de la información estadística a través de la constitución de redes facilita y simplifica enormemente este proceso. Podemos diferenciar tres tipos de redes de información estadística: productoras de información, intermediarias y redes de usuarios. Las redes productoras de información, en la actualidad con acceso on-line y sitio web propio, se caracterizan por su labor de producción integrada. Las redes intermediarias reelaboran y tratan la información que produce la primera tipología de redes y se especializan en la búsqueda de datos y los enlaces o vínculos a otras redes. Finalmente, las redes de usuarios se refieren a empresas, equipos investigadores. Respecto a las redes de intermediarios, EDA (Economic Development Administration) señala: "los intermediarios de datos tienen la función primaria de proporcionar accesos rápidos y de bajo coste a datos provenientes de diferentes fuentes, así como ayuda especializada en la elección y uso de la información”. La conexión entre los distintos tipos de redes se resume en el proceso que se sigue desde el nivel inicial que constituye la producción de información hasta la difusión y posterior utilización de la misma. La difusión de la información puede realizarse por el propio productor de la misma o a través de distribuidores intermediarios.

Lo cierto es que no es lo mismo producir información estadística, lo que denominamos en forma genérica datos o series temporales en economía, que utilizarla. De igual forma, desde la investigación puede llegarse a la innovación tras su correspondiente desarrollo, pero, evidentemente, investigación no coincide exactamente ni necesariamente con innovación.

En el ámbito concreto de la predicción económica, poder predecir la evolución de una economía para anticiparse a la toma de decisiones o a la aplicación concreta de políticas económicas es un deseo inherente a todos los analistas económicos, ya sean particulares o institucionales. A tal efecto, se han desarrollado muchos modelos matemáticos y econométricos con el fin de ayudar en este proceso. Lo habitual es trabajar con series temporales de baja frecuencia 
(datos anuales) o de alta frecuencia (datos trimestrales, mensuales) como es el caso de los indicadores de coyuntura económica. En este último caso, la irrupción del big data, macrodatos o datos masivos, con la implicación que conlleva referida al almacenamiento de un número elevado de datos y a los procedimientos desarrollados para encontrar en ellos patrones repetitivos de comportamiento, ha supuesto un cambio considerable en el paradigma económico. Aún de menor aplicación o desarrollo en las variables económicas tradicionales, pero con un despliegue importante en la economía de la salud o del turismo, por ejemplo.

Como paso previo, la selección de indicadores económicos y su traslación a indicadores compuestos de actividad económica ha supuesto un importante avance en el conocimiento económico. Se han definido distintas tipologías de indicadores a partir de la observación del comportamiento de series temporales de índole económica. Así, podemos referirnos a indicadores adelantados, coincidentes o retrasados. Los indicadores adelantados proporcionan señales sobre el comienzo de un nuevo ciclo económico y pueden ser incluso representativos de un cambio de ciclo. Algunos indicadores presentan una evolución que coincide con el patrón de comportamiento del ciclo económico en curso, confirmando la situación descrita y cuya evolución permite inferir información sobre la profundidad del ciclo analizado. Los indicadores retrasados se producen poco después de un periodo de expansión o decrecimiento económico y son útiles a la hora de determinar su extensión, pero también el cambio o el encadenamiento de ciclos. A partir de estos indicadores se construyen los denominados indicadores compuestos de actividad económica. La experiencia internacional en la elaboración de indicadores sintéticos, habitualmente de frecuencia trimestral y menor en el caso mensual, es muy amplia. La historia de los indica-dores cíclicos de actividad económica se inicia a finales de la década de los años 30 y principios de los años 40 cuando el National Bureau of Economic Research (NBER) publica el trabajo de Burns y Mitchell (1946) referido a la relación de indicadores coincidentes, rezagados y adelantados del ciclo económico de Estados Unidos. Como exponen Lahiri y Moore (1991), este trabajo previo de Burns y Mitchell se ha utilizado con éxito en diferentes países desde entonces configurándose como una de las herramientas predictivas que permite un enfoque menos restrictivo de la teoría sin renunciar a la amplitud y robustez de los resultados. En los nuevos desarrollos, subyace, en todo caso, la formulación propuesta en su momento por Lucas $^{2}$ (1977) referida a que "el ciclo económico no se refiere a una serie en particular, más bien el ciclo económico se refiere al comportamiento dinámico y la interacción entre distintas variables económicas”.

\footnotetext{
${ }^{2}$ Robert E. Lucas, Premio Nobel de Economía en 1995 por "haber desarrollado y aplicado la hipótesis de las expectativas racionales y haber transformado el análisis macroeconómico y profundizado la comprensión de la política económica”.
} 
En diversas publicaciones de la Organización para la Cooperación y el Desarrollo Económico, OCDE, (1999, 2010) pueden encontrarse referencias metodológicas para la construcción de indicadores compuestos construidos a partir de la información procedente de indicadores individuales (series temporales de datos) con diferentes niveles de agregación. También en Guidetti y Gyomai (2012) se encuentran referencias más cercanas al sistema actual de indicadores compuestos adelantados de la OCDE. Respecto a la aplicación concreta a otras áreas geográficas las referencias bibliográficas son múltiples y una simple búsqueda en internet nos permite encontrar la información deseada (más de 300.000 referencias disponibles para "indicadores compuestos de actividad económica”).

Los indicadores compuestos de actividad económica, además de informar sobre la evolución de una economía en particular, posibilitan aproximarse e incluso anticiparse al comportamiento del producto interior bruto (PIB). Lo cierto es que para valorar el estado o situación de una economía y sus perspectivas de evolución a futuro, lo habitual es centrarse en las predicciones de crecimiento del PIB, como medida comparativa de la situación económica de diferentes países.

Precisamente, en este trabajo reflexionamos sobre la creación de conocimiento económico a través de las predicciones de crecimiento del PIB como instrumento para extrapolar el comportamiento futuro de una economía. El proceso de elaboración del PIB comienza con toda la información estadística que integra la magnitud correspondiente, elaborada por los Institutos Nacionales de Estadística. Podría contemplarse el PIB sólo como información ya generada a partir de los datos previos que lo integran. Aunque también es cierto que incluso el valor del PIB en sí mismo es un dato, que ha de ser convenientemente interpretado para convertirse en información y después generar el conocimiento preciso sobre la evolución y situación de la economía a la que se refiere. Un paso adicional lo conforma la posibilidad de realizar estimaciones de evolución futura del PIB, predicciones o pronósticos que ayuden a la toma de decisiones en el ámbito económico-empresarial. En el siguiente epígrafe nos referiremos al proceso de predicción general en el ámbito de la economía, destacando algunas limitaciones y ventajas. A continuación, nos centraremos en las predicciones de crecimiento de la actividad económica regional en España, medidas por la proyección a futuro del PIB regional que realizan varias instituciones de forma periódica. Pretendemos orientar al usuario de predicciones económicas regionales sobre la conveniencia de utilizar una fuente en particular por su mayor fiabilidad o recurrir a la estimación de consenso. Finalmente, en el último apartado se presentan las principales conclusiones obtenidas. 


\section{EL PROCESO DE PREDICCIÓN EN ECONOMÍA}

El interés por la predicción en economía, fundamentada en diversas técnicas y modelos, además de recogerse en numerosas publicaciones al respecto, ha tenido su reconocimiento académico, siendo galardonados con el Premio Nobel de Economía diversos autores por sus contribuciones ${ }^{3}$. En 1969, Klein ${ }^{4}$ exponía el auge de la modelización econométrica y la incipiente preocupación por la obtención de predicciones referidas a ámbitos territoriales distintos del habitual vinculado a países o grandes áreas geográficas: "hay una petición interminable de usuarios de modelos para obtener más detalles”.

El problema de la elección y la ventaja de contar con predicciones en España ya quedaba recogido hace más de tres décadas en palabras de Espasa (1980) “en el mundo económico se plantea con frecuencia el problema de elegir entre alternativas diversas sin conocer con exactitud cuáles serán sus efectos, ya que éstos dependen de hechos que son desconocidos a la hora de tomar la decisión. A menudo la forma de actuar ante tal situación consiste en que el agente económico se forma una idea de cómo ocurrirán los hechos que desconoce y decide en consecuencia”. Este modo de proceder para predecir el futuro resultaba generador de inconvenientes para el autor, pues las percepciones sobre lo que va a suceder dificultan el proceso de evaluación de éxitos y de aprendizaje de los errores cometidos o fracasos: "el desarrollo de métodos de predicción transmisibles, intercambiables y evaluables es un área de investigación de gran importancia actual”.

A comienzos de los años noventa, destacaba Bolton (1991) el aumento del interés por los modelos macro-econométricos regionales y por su empleo con fines predictivos. En la actualidad, los avances en el campo de las tecnologías de la información, el procesamiento de los datos y el desarrollo de programas informáticos específicos han posibilitado un progreso notable de las técnicas y modelos aplicados a la predicción. La disponibilidad de una información consistente, de fácil disposición y rápida actualización, facilita el proceso. Ahora bien, a medida que recopilamos más datos y de diferentes tipos, a menudo encontramos que "simplemente tenemos más que explicar y que nuestra comprensión del comportamiento económico no mejora, necesariamente, con grandes conjuntos de datos o incluso mejores” (Phillips, 2004).

\footnotetext{
${ }^{3}$ El Premio Nobel de Economía (Sveriges Riksbank Prize in Economic Sciences in Memory of Alfred Nobel) se concede desde 1969, vinculado en numerosas ocasiones a los trabajos relativos a la conexión entre modelos teóricos y modelos empíricos. En el ámbito del desarrollo de la modelización aplicada a la economía encontramos a los galardonados R. Frisch y J. Tinbergen, 1969, L.R.Klein, 1980, T. Haavelmo, 1989, R.F. Engle y C. Granger, 2003.

${ }^{4}$ Lawrence R. Klein, Premio Nobel de Economía en 1980 por "la creación de modelos econométricos y la aplicación al análisis de las fluctuaciones económicas y políticas económicas”.
} 
Si es complicada la modelización económica más aún lo es la predicción. La principal cuestión a sopesar en el proceso de predicción económica, sea cual sea la variable elegida, es la consideración de la ciencia económica en su adecuado contexto. La economía no es una ciencia exacta, es una ciencia social que depende de las relaciones entre sus agentes sociales. Por ello, el futuro económico es incierto y viene determinado por el comportamiento humano y las decisiones futuras. Y a pesar de la extensa y creciente literatura sobre los ciclos económicos, la proyección de variables económicas nunca será una ciencia exacta. Aunque los economistas han mostrado con cierta claridad las causas y consecuencias de la reciente recesión global y la recuperación subsecuente, nuestra comprensión de por qué tienen lugar estas situaciones aún sigue siendo limitada (Kose y Terrones, 2015).

Loungani (2001) recoge un estudio sobre la exactitud de las previsiones económicas de crecimiento del PIB real en un número amplio de países industrializados en la década de los noventa. Concluye respecto al fracaso generalizado de predecir recesiones y señala que existe un alto grado de similitud entre las previsiones de crecimiento del sector privado y de las organizaciones internacionales (FMI, la OCDE y el Banco Mundial). El trabajo también proporciona evidencia sobre la eficiencia y el grado de sesgo de las previsiones económicas.

Para Hong (2006) una limitación básica se debe al hecho de que cualquier economía no es un experimento controlado. Inconveniente que se extiende al análisis económico como un todo: "las relaciones económicas son a menudo cambiantes en el tiempo para una economía, las variaciones de régimen y los cambios estructurales son más bien una regla que una excepción, debido a los choques tecnológicos y los cambios en las preferencias, estructura de la población y a las disposiciones institucionales”. Centrándonos en los modelos económicos globales y sus resultados en términos operativos, Pulido (2015) señala "parece que, en ocasiones, los modelizadores de la economía mundial no han valorado adecuadamente la complejidad de la tarea y el coste de mantener este tipo de modelos".

Kantorovich $^{5}$ (1975) ya manifestaba que "la etapa más dura a la hora de construir un modelo es la obtención de datos, ya que, al no haberlos necesitado nadie anteriormente, pueden contener errores sustanciales o incluso no existir. Hay dificultades de principio en la predicción de datos futuros y en las previsiones de desarrollos industriales”. Refiriéndose en particular al ámbito de la economía sostenía que "el hecho económico es difícil de describir formalmente, dada su complejidad y peculiaridad. Los modelos ahondan tan sólo en algunos de sus aspectos y sólo describen la realidad económica de forma aproximada y

\footnotetext{
${ }^{5}$ Leonid Vitálievich Kantoróvich recibió en 1975 el Premio Nobel de Economía, junto a Tjalling Koopmans, por "su contribución al desarrollo de métodos para el análisis de problemas económicos referidos a la asignación óptima de recursos escasos”.
} 
ruda, por lo que es generalmente difícil estimar la corrección de sus descripciones y sus conclusiones”. Aunque Kantorovich señalaba estas dificultades, y otras, confiaba en las perspectivas de propagación de los métodos matemáticos en la ciencia económica.

Pese al paso del tiempo y a la sustancial mejora de las condiciones en que se obtiene la información y se procesa, algunas de las dificultades señaladas no han podido ser aún soslayadas. No obstante, es preciso reconocer que esta circunstancia no resta importancia a la necesidad de continuar avanzando en el proceso de convertir la información en conocimiento. Recurriendo a los clásicos, para enfatizar la importancia de la predicción económica, Friedman (1953) establecía en su metodología de la economía positiva que "el objetivo último de una ciencia positiva es el desarrollo de una teoría o hipótesis que genere predicciones válidas y significativas sobre fenómenos que todavía no se han observado". Al respecto, más recientemente, Piffano (2012) señala que "habrá de reconocerse entonces que en las ciencias sociales [como es la economía] esas premisas básicas del análisis positivo en las cuales se habrán de basar los paradigmas, implican cierta subjetividad del analista, quien intentará describir los hechos y eventualmente pronosticar sus implicaciones, en una constante labor de corroboraciones provisorias y refutaciones”. Básicamente, lo que subyace es la reflexión en torno a que la interpretación de los hechos (que pueden ser los datos) en una ciencia social, como lo es la economía, depende del sujeto o agente que los interpreta, algo que no sucede en las ciencias empíricas.

En cualquier ámbito, la predicción siempre es una tarea compleja sujeta a múltiples condicionantes e hipótesis y, como tal, no exenta de riesgos. Como afirman Pulido y López (1999) "la predicción económica exige en la mayoría de las ocasiones el empleo de técnicas/modelos estadístico-econométricas para garantizar cierta fiabilidad en el proceso".

La fiabilidad de las predicciones viene condicionada por la metodología utilizada en el proceso de predicción y por el tipo de información disponible. Para valorar o incluso cuantificar la fiabilidad se recurre a criterios de diversa índole. Por un lado, los criterios económicos que vendrán determinados por la magnitud obtenida en los datos predichos y, por otro, los criterios estadísticos que se basan en medidas de aproximación al dato real.

Es importante considerar que la publicación y difusión de predicciones de crecimiento económico puede repercutir en el funcionamiento de los mercados financieros, cuestionar o apoyar las políticas gubernamentales o incluso influir en las decisiones o comportamiento de los agentes económicos. Todas estas posibles incidencias repercuten de nuevo en la influencia del propio futuro que anticipan como si de un bucle se tratase. Todo ello genera conocimiento, por eso es importante valorar la fiabilidad de la predicción. 
En el ámbito de la elaboración de predicciones de crecimiento económico, algunas pueden realizarse por aproximación o incluso por cierta intuición (habitual en el ámbito empresarial), mientras que otras pueden basarse en el análisis de indicadores, recoger datos de encuestas especializadas o aplicar técnicas y modelos de computación más o menos sofisticados.

En el proceso de predicción económica, el núcleo lo constituye la moderna metodología econométrica, incorporando en ella tanto los desarrollos en el tratamiento de series temporales, la econometría espacial e incluso la información subjetiva a través de variables de expectativas, restricciones sobre parámetros o factores adicionales a considerar en la predicción.

Como señala Pulido (2006) "una de las alteraciones más importantes, con efectos que aún pueden prolongarse a futuro, es el paso de un enfoque restrictivo de la predicción a otro más flexible y prospectivo”. El enfoque restrictivo (predicción tradicional) permite obtener, de forma lo más automática posible, una predicción no contaminada por opiniones y criterios personales. Se basa en disponer de esquemas de conjunto perdurables en el tiempo, que manejan una amplia información estadística de tipo cuantitativo. En un enfoque amplio (predicción prospectiva), como corresponde a un proceso de predicción dinámico y global, los aspectos cuantitativos conviven con los cualitativos. Un complejo sistema de predicción combina diversos ámbitos (internacional, nacional, regional y sectorial), con distintas periodicidades (anuales, trimestrales y mensuales), diferentes técnicas (opiniones de expertos, series temporales, modelos) y fuentes externas de predicción o incluso redes. El predictor puede utilizar diferentes técnicas y modelos de predicción para desarrollar su propia apuesta de futuro. El usuario de las predicciones, que no siempre coincide con el predictor, tiene la posibilidad de combinarlas de forma que mejor se ajusten a sus intereses de conocimiento económico.

No obstante, pese a las mejoras observadas en las técnicas y modelos econométricos, bases de datos de mayor amplitud y de mejor calidad, debemos considerar siempre los riesgos del proceso de predicción. De hecho, una cuestión fundamental, que se apoya sobre todo análisis económico práctico, es el grado de certeza en que podemos esperar entender los fenómenos económicos en el proceso de desarrollo a partir de una teoría. La recogida de los datos, su correcta interpretación, para ser considerada como información relacionada y el posible ajuste de un modelo empírico para alcanzar el grado de conocimiento preciso completan la sucesión. Como señala Phillips (2004) “cuarenta años de experiencia empírica en previsión macroeconómica sugieren que hay límites a nuestra capacidad de hacer predicciones sobre la actividad económica”.

Respecto a los posibles fallos en las predicciones económicas, Rickman (2014) discute "el potencial para un mayor uso de una nueva generación de modelos macroeconómicos en predicción regional y análisis cuantitativo de 
políticas”. Refiriéndonos a la utilidad de las predicciones económicas, coincidimos con las apreciaciones de Pulido (2006) "aún con todos sus errores potenciales, la predicción supone una referencia imprescindible de futuro; la mayor o menor discrepancia entre predicciones diversas indican el grado de acuerdo o riesgo que un usuario tiene de aceptar cualquiera de ellas; la revisión periódica de las predicciones es un buen indicador de la mejora o empeoramiento de las expectativas de los expertos”. Siguiendo a Graefe (2015), las personas y las organizaciones comúnmente toman decisiones combinando la información de múltiples entradas, y en ese proceso la predicción es útil.

\section{PREDICCIONES DE CRECIMIENTO DE LA ACTIVIDAD ECONÓMICA REGIONAL EN ESPAÑA}

\subsection{Consideraciones sobre el PIB como medida de la actividad económica}

El PIB es el principal y habitual instrumento de medida de la actividad económica desarrollada dentro de un país, sin descontar los consumos de capital empleados. El PIB se ha adoptado como medida universal comparable de la actividad económica entre distintas zonas geográficas o territoriales. Precisamente, en este artículo que reflexiona sobre el proceso en que los datos se convierten en información y ésta en conocimiento, tiene mayor sentido incluso poner de manifiesto algunas inquietudes en torno a la medición del PIB. Su cálculo se rige por normas internacionales basadas en el trabajo previo de reflexión que define sus bases estadísticas y conceptuales. Calcular todas las actividades económicas de un país, en definitiva cuantificar monetariamente todos los bienes y servicios producidos, es un proceso que entraña diversas dificultades. No todo se mide y no todo puede medirse, sin entrar ahora en las disquisiciones propias del cómputo de la economía sumergida y su incorporación en el PIB (en España representa casi el 20\% del PIB, Arrazola et al., 2011).

No obstante, diversos autores han señalado las limitaciones que presenta el PIB, especialmente en su faceta de indicador de funcionamiento económico y progreso social. Ya en 1987, Solow ${ }^{6}$ concluía su reseña de un ensayo sobre la economía post-industrial con una frase bien concisa: "la era de los ordenadores puede verse por doquier, excepto en las estadísticas de productividad”. A esta misma apreciación se han sumado Stiglitz y Sen ${ }^{7}$. Como plantean Stiglitz et al. (2008) “el PIB no es erróneo en sí, sino que se emplea de forma errónea. El problema surge cuando se utiliza para aproximarse a conceptos que no puede

\footnotetext{
${ }^{6}$ Robert Merton Solow, Premio Nobel de Economía en 1987 por “sus contribuciones a la teoría del crecimiento económico".

${ }^{7}$ Joseph Eugene Stiglitz, Premio Nobel de Economía en 2001, junto con George Akerlof y Michael Spence, por "sus análisis de los mercados con información asimétrica". Amartya Sen, Premio Nobel de Economía en 1998 por "sus contribuciones a la economía del bienestar”.
} 
abarcar en su complejidad como es el bienestar social, económico, el progreso, calidad de vida, la desigualdad,...”. El PIB tiene ciertas limitaciones asumidas para evaluar el bienestar a lo largo del tiempo, en particular en sus dimensiones económica, medioambiental y social, algunos de cuyos aspectos se suelen designar con el término de sustentabilidad o sostenibilidad.

En un enfoque más amplio suele atenderse al binomio PIB/Empleo para referirse al crecimiento económico. En España se dispone de revisiones anuales, trimestrales e incluso mensuales de las principales variables macroeconómicas nacionales realizadas por más de veinte instituciones públicas o privadas, detrás de las que están equipos consolidados de trabajo. A ello se une el amplio desarrollo de las mejoras en la realización y uso de predicciones económicas, con la publicación de datos oficiales cada vez más fiables y de más rápida difusión, y una mayor rapidez en la revisión de predicciones, especialmente en entornos de riesgos e incertidumbre. Las nuevas técnicas disponibles se han visto igualmente reforzadas gracias a un tratamiento informático más flexible y potente. Diebold (1997) ya establecía que el "sello distintivo de la previsión macroeconómica en los próximos veinte años será la alianza de lo mejor de los enfoques no estructurales y estructurales, facilitada por los avances en las técnicas numéricas y de simulación que ayudarán a los macroeconomistas para resolver, estimar, simular y predecir con modelos fructíferos”.

Como ya se ha señalado, la primera premisa que tenemos que considerar en el ámbito de la predicción económica es que la economía no es una ciencia exacta, es una ciencia social, donde el comportamiento de sus agentes (hogares, empresas, gobierno) condiciona la evolución de cualquier magnitud. Y ese comportamiento no es predecible, ni tampoco fácilmente aproximable, al estar afectado por decisiones que se toman en función de la información y otros factores difíciles de cuantificar, como son las expectativas o la percepción. Precisamente, la percepción económica puede ser un buen indicador de lo que se espera ocurra en la evolución del PIB. Por ejemplo, en Baker et al. (2015) proponen la elaboración de un índice de incertidumbre de política económica Economic Policy Uncertainty Index para quince países o áreas geográficas, incluido España (los datos mensuales pueden encontrarse en www.policyuncertainty. com). El índice de incertidumbre en la política económica se construye a partir de tres tipos de componentes subyacentes: cobertura periodística de la incertidumbre relacionada con la política económica, disposiciones relacionadas con tributos que expirarán en los próximos años y el grado de desacuerdo entre los analistas económicos. En el caso de España, la evolución del índice refleja unos niveles actuales de incertidumbre de política económica sumamente elevados comparados con la historia reciente. Un aumento de la incertidumbre de política económica presagia una disminución en el crecimiento económico, en términos de PIB, y el empleo en los meses siguientes. En Alvira y García (2000) utilizan datos de una encuesta del Centro de Investigaciones Sociológicas (CIS, en- 
cuesta 2286/98 sobre instituciones y autonomías) para comparar la situación económica según algunos datos objetivos (renta por habitante, tasa de paro) con la situación percibida por los ciudadanos. Concluyen que "en ocasiones aparecen discrepancias importantes entre la percepción social y los indicadores objetivos”. Algunos factores explicativos de estas diferencias son la insularidad y el alejamiento geográfico de la península, el peso de la economía sumergida, el recuerdo de algún sector industrial pujante en épocas pasadas.

En el proceso para obtener predicciones de crecimiento regional juega un papel importante la información previa, los datos y variables que constituyen las series temporales de partida. La demanda de información regional referida a los datos de producción regional, ya sea PIB o valor añadido bruto, VAB, puede ser parcialmente satisfecha con la información de carácter anual que contiene la Contabilidad Regional de España (CRE) que publica el Instituto Nacional de Estadística (INE). La CRE recoge el sistema de Cuentas Regionales de España, según la metodología establecida en el Sistema Europeo de Cuentas Nacionales y Regionales SEC-2010. Ofrece una descripción cuantificada, sistemática y lo más completa posible de la actividad económica regional en España (comunidades autónomas y provincias) durante el período de referencia considerado, analizada tanto desde el enfoque funcional como desde el enfoque institucional (www.ine.es). En este trabajo nos centramos en el análisis del crecimiento de la actividad económica regional en España a partir de las predicciones de evolución del PIB regional realizadas por diferentes instituciones, sustentadas en los datos de la CRE, cuestión que abordamos a continuación.

\subsection{Experiencias en predicciones de crecimiento económico regional en España}

Para el ejercicio que nos ocupa, podemos recurrir en la actualidad a diferentes instituciones públicas y privadas que proporcionan predicciones del PIB o del VAB regional. La comparativa de predicciones alternativas de crecimiento económico regional requiere centrarnos sólo en las aportaciones que proporcionan predicciones para todas las comunidades autónomas a la vez, y no para una sola en particular, congruentes con un marco nacional y con cierta periodicidad regular. Con estas premisas, las instituciones seleccionadas son:

AIReF: Autoridad Independiente de Responsabilidad Fiscal, creada por Ley Orgánica 6/2013, de 14 de noviembre, para reforzar "el compromiso de lograr un control eficaz del cumplimiento de los objetivos de estabilidad presupuestaria y deuda pública”. www.airef.es.

BBVA Research: Departamento que "adapta el análisis económico a las necesidades de un grupo bancario recientemente internacionalizado, para responder al reto de evaluar desarrollos económicos cada vez más globalizados”. 
Desarrollan una amplia labor en el ámbito de la predicción económica que difunden con documentos de trabajo, notas de prensa. www.bbvaresearch.com.

CEPREDE: Centro de Predicción Económica ubicado en la Universidad Autónoma de Madrid dedicado a la investigación económica, constituido como asociación sin ánimo de lucro. En sus informes mensuales y semestrales recogen la evolución de las principales magnitudes macroeconómicas, incorporando estimaciones e indicadores que permiten adelantar la evolución de la situación económica en el futuro. www.ceprede.es.

FUNCAS: Institución de carácter privado, sin ánimo de lucro, creada y financiada por la CECA dentro de su Obra Social "para el desempeño de actividades que redunden en beneficio de la sociedad española, promuevan el ahorro y contribuyan a extender el conocimiento de las cajas de ahorros facilitando su servicio a la sociedad”. Es una institución activa en la promoción y difusión de estudios e investigaciones económicas y sociales. www.funcas.es.

HISPALINK: red de equipos universitarios dedicada a la modelización regional integrada, cuyo objetivo principal es la revisión y análisis de la situación actual y perspectivas económicas de las regiones españolas. www. hispalink.es.

Tabla 1

Fuentes alternativas para la obtención de predicciones de crecimiento del PIB regional

\begin{tabular}{|c|c|c|c|c|c|}
\hline Instituciones & AIReF & $\begin{array}{c}\text { BBVA } \\
\text { Research }\end{array}$ & CEPREDE & FUNCAS & HISPALINK \\
\hline $\begin{array}{l}\text { Referencias } \\
\text { metodológicas }\end{array}$ & AIREF (2015) & $\begin{array}{l}\text { Camacho y } \\
\text { Domenech } \\
\quad(2010)\end{array}$ & $\begin{array}{l}\text { Meriguete y } \\
\text { Pérez (2014) }\end{array}$ & $\begin{array}{c}\text { Alcaide, } \\
(1996), \\
\text { Alcaide y } \\
\text { Alcaide }(2000)\end{array}$ & $\begin{array}{l}\text { Pérez et al. (2009), } \\
\text { Callealta y López } \\
\text { (2001) }\end{array}$ \\
\hline $\begin{array}{l}\text { Síntesis metodología } \\
\text { empleada }\end{array}$ & $\begin{array}{l}\text { Indicador } \\
\text { sintético de } \\
\text { actividad } \\
\text { trimestral para } \\
\text { cada CCAA }\end{array}$ & $\begin{array}{l}\text { Indicador } \\
\text { sintético de } \\
\text { actividad } \\
\text { trimestral para } \\
\text { cada CCAA }\end{array}$ & $\begin{array}{l}\text { Indicador } \\
\text { sintético de } \\
\text { actividad } \\
\text { mensual para } \\
\text { cada CCAA }\end{array}$ & $\begin{array}{l}\text { Indicadores y } \\
\text { modelos para } \\
\text { cada CCAA }\end{array}$ & $\begin{array}{c}\text { Modelos } \\
\text { econométricos } \\
\text { unirregionales y } \\
\text { Modelo } \\
\text { multirregional para } \\
\text { la congruencia }\end{array}$ \\
\hline $\begin{array}{l}\text { Equipos } \\
\text { especializados en } \\
\text { predicción regional }\end{array}$ & 1 equipo & $\begin{array}{c}1 \text { equipo (unidad } \\
\text { de economía } \\
\text { española) }\end{array}$ & $\begin{array}{c}1 \text { equipo } \\
\text { universitario }\end{array}$ & 1 equipo & $\begin{array}{c}16 \text { equipos } \\
\text { universitarios y } 1 \\
\text { equipo que coordina }\end{array}$ \\
\hline $\begin{array}{l}\text { Prioridad enfoque } \\
\text { nacional/regional }\end{array}$ & Top-down & Top-down & Top-down & Top-down & Bottom-up \\
\hline $\begin{array}{l}\text { Periodicidad de la } \\
\text { predicción/revisión }\end{array}$ & Trimestral & Semestral & Mensual & Semestral & Semestral \\
\hline $\begin{array}{l}\text { Frecuencia temporal } \\
\text { de los datos de } \\
\text { predicción }\end{array}$ & Trimestral & Anual & Mensual & Anual & Anual \\
\hline $\begin{array}{l}\text { Detalle sectorial de la } \\
\text { predicción }\end{array}$ & No & No & No & Sí (4 ramas) & Sí (4 ramas) \\
\hline $\begin{array}{l}\text { Horizonte temporal } \\
\text { de la predicción }\end{array}$ & $\begin{array}{c}\text { Trimestre } \\
\text { cerrado por } \\
\text { Contabilidad } \\
\text { Nacional } \\
\text { Trimestral }\end{array}$ & $\begin{array}{l}\text { Año en curso y } \\
\text { siguiente }\end{array}$ & $\begin{array}{l}\text { Meses del } \\
\text { año en curso }\end{array}$ & Año en curso & $\begin{array}{l}\text { Año en curso y dos } \\
\text { años siguientes }\end{array}$ \\
\hline
\end{tabular}

Fuente: Elaboración propia a partir de las instituciones señaladas. 
En la Tabla 1 resumimos los principales aspectos del proceso de predicción regional y en las referencias bibliográficas puede encontrarse un mayor detalle sobre la metodología empleada por cada institución.

En general, las cinco fuentes consultadas coinciden en la combinación de tres tipos de información estadística, de índole coyuntural y estructural. Por un lado, los datos mensuales de indicadores de coyuntura económica desagregados a nivel regional, que algunas emplean para la construcción de un indicador sintético o compuesto de actividad económica regional para distribuir el PIB nacional, y otras como fuente de alimentación de modelos econométricos regionales. Respecto a los indicadores seleccionados, en el primer caso son comunes a todas las CC.AA., mientras que en el segundo cada modelo regional utiliza la información que considera recoge de forma más fiel la evolución de su estructura productiva regional. En segundo término, emplean los datos anuales que recoge la CRE para las series históricas oficiales de evolución del PIB regional $\mathrm{y}$, finalmente, las estimaciones de crecimiento económico trimestral nacional elaboradas en la Contabilidad Nacional Trimestral (CNTR) del INE para asegurar la consistencia de las estimaciones regionales individuales.

La obtención de las predicciones puede realizarse por la aplicación de procedimientos de estimación arriba-abajo, top-down, que otorga primacía a la distribución del dato nacional por regiones o abajo-arriba, bottom-up, que implica obtener el dato nacional por agregación de los datos regionales. En López (2015) se describen los distintos enfoques en la modelización regional (unirregional, multirregional) y los procedimientos de estimación señalando que "hay una tendencia para apoyar los modelos top-down debido a sus menores costes de desarrollo, en términos de coste-eficacia de los resultados”. No obstante, los modelos top-down no permiten la retroalimentación: la vuelta de la región a la nación o entre regiones, principal ventaja de los modelos bottom-up (Klein, 1969).

A la hora de predecir una variable económica, como es el PIB, a partir de la información conjunta que aporta una selección previa de variables explicativas (indicadores o datos) hay diversas consideraciones. La selección previa de variables relevantes se basa en criterios de aproximación al estudio de la variable que queremos predecir, ya sea por su elevada correlación o proximidad conceptual. En la práctica encontramos dos tendencias contrapuestas. Por un lado, la que aboga por utilizar un modelo que combine toda la información disponible (combination of information, CI). Por otro, la que apuesta por utilizar la metodología de combinación de pronósticos (forecast combination, FC). Hay diversas opiniones respecto a si el pronóstico generado por toda la información incorporada en un solo paso, CI, y, por tanto, más laborioso, es mejor o no que la combinación de las predicciones de los modelos individuales donde cada uno incorpora información parcial, FC. 
Engle, Granger y Kraft (1984) señalaban: "la mejor predicción se obtiene mediante la combinación de información ... Si se conocen los dos modelos, se debe combinar la información que entra en los modelos, y no las previsiones que salen de los modelos”. Diebold y Pauly (1990) apuntan "... hay que reconocer que en muchas situaciones de pronóstico, sobre todo en tiempo real, la puesta en común de los conjuntos de información es imposible o prohibitivamente costosa". Como afirman Huang y Lee (2007) "hasta donde sabemos, no hay ninguna prueba formal en la literatura para demostrar que CI es mejor que FC”.

En este sentido, al comparar las diferentes predicciones sobre el PIB regional, podemos decidir si es mejor decantarse por una sola de las fuentes, la que pueda considerarse de mayor fiabilidad, o bien recurrir a la predicción media, la que se denomina de consenso. Como indican Vicens y Medina (2014), al reflexionar sobre la "popularización" de las predicciones económicas en el sentido de facilidad de acceso a las mismas, se hace necesario "aportar datos sobre la fiabilidad de las predicciones dada la importancia y repercusión social y mediática que éstas tienen", pues pueden influir sobre el comportamiento económico de los agentes implicados. Los autores demuestran la existencia de un sesgo sistemático en las predicciones del PIB para España realizadas por organismos internacionales como la Comisión Europea, OCDE y el FMI . En periodos de crecimiento económico las predicciones tienen a infravalorar los datos reales mientras que durante el periodo de crisis se observa un sesgo al alza en los datos predichos. Pons (1999) estudia la calidad de las predicciones de crecimiento de siete magnitudes de la economía española, entre ellas el PIB. Compara las predicciones realizadas por el Ministerio de Economía y Hacienda para la elaboración del cuadro macroeconómico que acompaña a los Presupuestos Generales del Estado con las predicciones que realiza la OCDE en el periodo 1983-1997. Pons defiende la utilidad de la previsión económica y, en particular, la previsión de las principales magnitudes económicas de un país, pues muestran la senda de evolución a futuro y constituyen un apoyo a la toma de decisiones y a la implementación de políticas económicas alternativas. Concluye en su análisis que las predicciones estudiadas son racionales "puesto que a medida que se dispone de un volumen de información superior sobre las perspectivas futuras de la economía, aumenta la calidad de las predicciones económicas”.

En OCDE (2014) se analizan los errores de predicción del crecimiento económico en los países de la OCDE durante el periodo de crisis 2007-2012. En términos generales, el crecimiento del PIB fue sobrestimado, siendo el ritmo de crecimiento real más débil que el pronosticado, no sólo en el punto álgido de la crisis sino también en el periodo de recuperación subsecuente. La experiencia derivada del análisis realizado ha conducido a sucesivas revisiones posteriores de los pronósticos. Como era de suponer, los errores en las proyecciones de crecimiento del año corriente, medidos por el error cuadrático medio (root mean 
squared error), son menores que los correspondientes a las proyecciones de crecimiento del PIB para el año siguiente.

Con mayor frecuencia, la tendencia es decantarse por la predicción de consenso, en terminología anglosajona Blue chip average forecast o consensus forecast, basada en calcular el promedio de las predicciones realizadas por distintas instituciones o analistas profesionales del ámbito de la predicción. Se trata de una combinación de predicciones (FC) a las que puede incluso aplicarse alguna ponderación distinta, en lugar de considerar la participación de todas las instituciones o fuentes alternativas por igual.

La cuestión fundamental que subyace es considerar qué es mejor: centrarse sólo en una predicción (seleccionada por distintos criterios de fiabilidad o precisión) o continuar con el consenso o promedio. Todo ello sin obviar la problemática inherente, el hecho de que la previsión puede ser inexacta crea un dilema serio en la adopción de decisiones y elaboración de políticas (Makridakis et al., 2009).

\subsection{Comparativa de predicciones de crecimiento económico regional}

Al cierre de este artículo, se dispone de datos referidos a la serie de VAB 2010-2015 con Base 2010, siendo 2010-2013 datos calificados como "provisionales”, 2014 de "avance” y 2015 de "primera estimación” (publicados en marzo de 2016). La información, a pesar de la limitación consecuente de datos históricos por el reciente cambio de base contable, es muy valiosa para el análisis de las diferentes estructuras productivas, pero no posibilita un seguimiento más cercano en el tiempo, de cómo evoluciona cada economía regional, al no ofrecerse datos consolidados de evolución trimestral.

Al examinar las estimaciones entre las diferentes comunidades autónomas pueden extraerse conclusiones previas sobre las estructuras productivas y su repercusión en el PIB regional. De hecho, la especialización de la estructura productiva y empresarial determina que ascensos en sectores y actividades de menor presencia en una región contribuyan también en menor medida a su crecimiento global de lo que lo hacen en otros territorios. Es decir, la contribución de cada sector al crecimiento agregado une no sólo las dinámicas de la actividad sino la importancia relativa de la misma.

Los últimos datos publicados en la CRE referidos al año 2015, primera estimación de marzo de 2016, presentan un crecimiento nacional del 3,2\% en términos de variación del PIB. Todas las comunidades autónomas crecieron por encima del dato registrado para el PIB del conjunto de la Unión Europea de 28 miembros (UE-28), del 1,8\% según Eurostat, la Oficina Estadística de la Unión Europea (Eurostat news release euro-indicators, febrero 2016), y con tasas superiores a la media del periodo 2001-2014 (Tabla 2). Las regiones líderes en crecimiento económico en 2015 (Comunidad Valenciana y Comunidad de Madrid) 
experimentaron un mejor comportamiento de la industria y la construcción, comparado con el promedio nacional.

\section{Tabla 2}

Evolución histórica del crecimiento del PIB regional en la CRE-INE (tasas de variación real)

\begin{tabular}{|l|c|c|c|c|c|}
\hline \multirow{2}{*}{ CC.AA. } & $\begin{array}{c}\text { Situación previa } \\
\text { de la crisis }\end{array}$ & Periodo crisis & Salida crisis & $\begin{array}{c}\text { Promedio periodo } \\
\text { completo }\end{array}$ & Salida crisis \\
\cline { 2 - 6 } & $\mathbf{2 0 0 1 - 2 0 0 7}$ & $\mathbf{2 0 0 8 - 2 0 1 3}$ & $\mathbf{2 0 1 4}$ & $\mathbf{2 0 0 1 - 2 0 1 4}$ & $\mathbf{2 0 1 5}$ \\
\hline Andalucía & 3,8 & $-1,6$ & 1,0 & 1,3 & 3,2 \\
\hline Aragón & 3,8 & $-1,3$ & 1,4 & 1,5 & 2,7 \\
\hline Asturias & 3,0 & $-2,1$ & 0,5 & 0,6 & 3,1 \\
\hline Baleares & 2,2 & $-1,0$ & 1,2 & 0,8 & 3,2 \\
\hline Canarias & 3,1 & $-1,2$ & 1,7 & 1,2 & 2,8 \\
\hline Cantabria & 2,8 & $-2,1$ & 1,0 & 0,5 & 2,6 \\
\hline Castilla y León & 2,8 & $-1,5$ & 1,0 & 0,9 & 2,9 \\
\hline Castilla-La Mancha & 4,7 & $-1,2$ & 0,3 & 1,8 & 3,2 \\
\hline Cataluña & 3,5 & $-1,5$ & 1,6 & 1,2 & 3,3 \\
\hline Com. Valenciana & 3,5 & $-2,0$ & 1,9 & 1,0 & 3,6 \\
\hline Extremadura & 3,5 & $-1,0$ & 1,3 & 1,4 & 3,0 \\
\hline Galicia & 3,7 & $-1,3$ & 0,4 & 1,3 & 3,2 \\
\hline Com. de Madrid & 4,0 & $-0,5$ & 1,6 & 1,9 & 3,4 \\
\hline Murcia & 4,1 & $-1,3$ & 1,8 & 1,6 & 3,1 \\
\hline Navarra & 3,3 & $-1,0$ & 1,6 & 1,3 & 2,9 \\
\hline País Vasco & 2,9 & $-1,2$ & 1,3 & 1,1 & 3,1 \\
\hline La Rioja & 3,5 & $-1,7$ & 2,0 & 1,2 & 2,8 \\
\hline ESPAÑA & 3,6 & $-1,3$ & 1,4 & 1,3 & 3,2 \\
\hline valor máximo & 4,7 & $-0,5$ & 2,0 & 1,9 & 3,6 \\
\hline valor mínimo & 2,2 & $-2,1$ & 0,3 & 0,5 & 2,6 \\
\hline
\end{tabular}

Fuente: Elaboración propia a partir de CRE-INE, con datos enlazados de serie contable SEC-1995 y SEC-2010.

La cuestión es que tendremos que esperar a marzo de 2017 para conocer el dato oficial de crecimiento regional en 2016 según la CRE-INE. Es por ello, que se precisa de la utilización de técnicas y modelos regionales para predecir las magnitudes económicas de una región concreta y poder avanzar en el conocimiento de la realidad económica. Podemos acometer el proceso de predicción u optar por recurrir a estimaciones realizadas por fuentes alternativas. Incluso en diciembre de 2016 se producirá la revisión oficial del dato de "primera estimación” de 2015 según la CRE de marzo de 2016, pasando a ser calificado como dato de "avance" y pueden generarse importantes cambios, como ocurrió previamente con el dato de 2014. En la Tabla 3 recogemos la diferencia de estimación del propio INE en el dato referido al año 2014, cuya "primera estimación" se produjo en la CRE de marzo de 2015, y revisión para pasar a ser dato "avance" en diciembre de 2015 hasta ser calificado más adelante como "provisional", paso previo a la situación de "definitivo". El INE señala que "las variaciones que se 
pueden observar respecto a las estimaciones de la serie anterior de la CRE-2010 publicadas en el mes de marzo pasado [2015] son debidas, tanto a la actualización de resultados de la Contabilidad Nacional de España como a la disponibilidad de una mayor cantidad de fuentes de información estadística”.

Tabla 3

Diferencias en la estimación del crecimiento del PIB del año 2014 en la CRE-INE, tasas de variación real. Datos publicados en diciembre 2015 "avance" vs. datos publicados en marzo 2015 "primera estimación"

\begin{tabular}{|l|c|c|c|}
\hline \multicolumn{1}{|c|}{ CC.AA. } & $\begin{array}{c}\text { CRE-PIB 2014 } \\
\text { dato “avance" } \\
\text { diciembre 2015 }\end{array}$ & $\begin{array}{c}\text { CRE-PIB 2014 } \\
\text { dato “1a estimación" } \\
\text { marzo 2015 }\end{array}$ & $\begin{array}{c}\text { Error de estimación } \\
\text { CRE-PIB 2014 }\end{array}$ \\
\hline Andalucía & 1,0 & 1,3 & $-0,3$ \\
\hline Aragón & 1,4 & 1,7 & $-0,3$ \\
\hline Asturias & 0,5 & 0,8 & $-0,3$ \\
\hline Baleares & 1,2 & 1,9 & $-0,7$ \\
\hline Canarias & 1,7 & 2,2 & $-0,5$ \\
\hline Cantabria & 1,0 & 1,0 & 0,0 \\
\hline Castilla y León & 1,0 & 1,4 & $-0,4$ \\
\hline Castilla-La Mancha & 0,3 & 1,2 & $-0,9$ \\
\hline Cataluña & 1,6 & 1,4 & 0,2 \\
\hline Com. Valenciana & 1,9 & 2,1 & $-0,2$ \\
\hline Extremadura & 1,3 & 2,2 & $-0,9$ \\
\hline Galicia & 0,4 & 0,5 & $-0,1$ \\
\hline Com. de Madrid & 1,6 & 1,0 & 0,6 \\
\hline Murcia & 1,8 & 2,0 & $-0,2$ \\
\hline Navarra & 1,6 & 2,0 & $-0,4$ \\
\hline País Vasco & 1,3 & 1,2 & 0,1 \\
\hline La Rioja & 2,0 & 2,5 & $-0,5$ \\
\hline ESPAÑA & 1,4 & 1,4 & 0,0 \\
\hline valor máximo & 2,0 & 2,5 & $-0,5$ \\
\hline valor mínimo & 0,3 & 0,5 & $-0,2$ \\
\hline
\end{tabular}

Fuente: Elaboración propia a partir de CRE-INE.

Para valorar la capacidad de la estimación (diferencia entre la primera estimación para 2014 realizada en marzo de 2015 y la revisión de diciembre de 2015) calculamos las medidas sobre los errores (Figura 2) como primer diagnóstico sobre la bondad de las estimaciones: RECM raíz del error cuadrático medio (root mean squared error) y PEAM porcentaje de error absoluto medio (mean absolute percentage error). En ambos casos, cuanto más cercanas estén a cero mejor será la capacidad predictiva del modelo (en este caso, mejor habrá sido la estimación del año 2014 realizada en primera instancia por la CRE-INE), siendo i: el número de casos a estudiar, las diecisiete comunidades autónomas más el dato nacional $(n=18) . Y_{i}$, en nuestro caso, es la observación referida a la estimación de crecimiento del PIB en 2014 realizada en diciembre de 2015. $e_{i}$ es la diferencia 
entre el dato "real" $\left(\mathrm{Y}_{\mathrm{i}}\right.$, ) y el dato "estimado" (PIB en 2014 publicado en marzo de 2015).

Figura 2

Medidas sobre los errores cometidos en la estimación

$$
R E C M=\sqrt{\frac{\sum_{i-1}^{n}\left(e_{i}-\bar{e}\right)^{2}}{n}} \quad P E A M=\frac{\sum_{i=1}^{n}\left|e_{i} / Y_{i}\right|}{n} * 100
$$

Pueden apreciarse variaciones significativas en el cálculo del error de estimación cometido, como diferencia entre el dato considerado como real ("avance": estimación de diciembre de 2015) y el dato estimado ("primera estimación": marzo 2015), superiores a medio punto en valor absoluto, en el caso de la estimación del año 2014 referido a Castilla-La Mancha, Extremadura, Baleares, Comunidad de Madrid y La Rioja, diferencias que conducen a porcentajes elevados de sus respectivos errores absolutos. En términos medios, considerando en conjunto todas las CC.AA, se obtiene un valor de RECM de 0,45 y una cuantificación de PEAM que indica que la estimación ha variado en un 43,5\%. Esta proporción refleja una diferencia considerable para tratarse de una revisión oficial de la estimación de un dato, especialmente porque, como tal, forma parte de la información incluida en los modelos e indicadores regionales para la elaboración de predicciones.

Figura 3

Diagrama "Predicción-Realización" de las diferencias de estimación del año 2014 en la CRE-INE. Datos publicados en diciembre 2015 "avance" vs. datos publicados en marzo 2015 "1ª estimación"

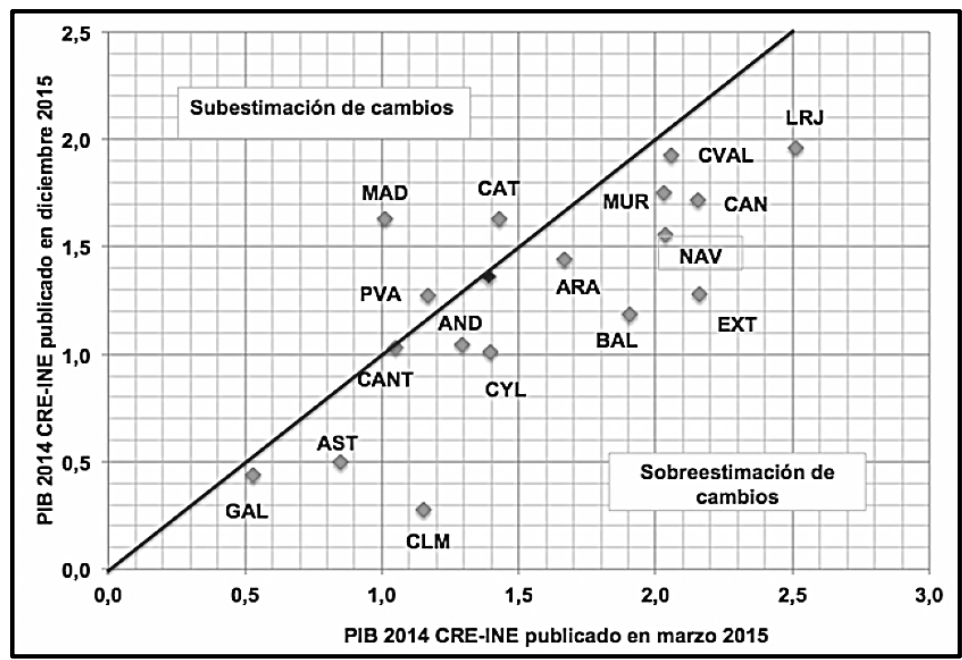

Fuente: Elaboración propia a partir de CRE-INE. 
Para analizar las características del error de estimación, elaboramos el diagrama de predicción-realización (Figura 3) propuesto por Theil (1961, 1966), donde se representan las tasas de crecimiento reales y estimadas (previstas), en nuestro caso para el crecimiento del PIB en 2014. La visión gráfica conjunta permite apreciar con mayor claridad las diferencias señaladas en términos de subestimación (infravaloración) y sobreestimación (sobrevaloración) y, por tanto, el grado de adecuación de la estimación realizada detectando los errores sistemáticos y los cambios de signo.

Con esta premisa de partida, procedemos ya a la comparativa del dato oficial de crecimiento del PIB regional en 2015 y las previsiones realizadas por las cinco fuentes seleccionadas (Tabla 4). En marzo de 2016 conocimos el dato referente al PIB de 2015 de cada una de las comunidades autónomas. Por ejemplo, la Comunidad Valenciana presentó un dato de 101.604 millones de euros, que se corresponde con un índice de volumen encadenado de 98,5. Son sólo datos que, de forma aislada, no aportan información relevante. Sin embargo, al calcular la tasa de crecimiento real, de un 3,6\% respecto a 2014 y comparar con la correspondiente al conjunto nacional $(3,2 \%)$ y la UE-28 $(1,8 \%)$ se extraen conclusiones relevantes respecto a una mayor dinamicidad del ritmo de crecimiento. Es extendido entre los analistas calificar crecimientos del PIB superiores al 3\% como expansión, entre el 2,1\% y 3\% como sostenido, entre 1\% y $2 \%$ debilidad. La comparativa con la situación de las demás comunidades autónomas permite comprobar que la Comunidad Valenciana lideró el crecimiento nacional en 2015, gracias al comportamiento de la industria manufacturera en la región. La combinación de las predicciones (consensus forecast) de cinco instituciones diferentes, realizadas unos meses antes de la publicación del dato oficial, situaba a la Comunidad Valenciana en segundo puesto, con una tasa de crecimiento del PIB del mismo orden.

Al tratarse de un año ya finalizado, la dispersión entre la predicción nacional y la que se corresponde con la agregación de predicciones regionales siempre se reduce a medida que nos acercamos al instante para el que se predice y con mayor consenso sobre lo que finalmente aconteció según los datos oficiales (aunque serán revisados en diciembre de 2016). Como también se expone en OCDE (2014), las diferentes predicciones de crecimiento presentan una mayor coincidencia al aproximarse al cierre del año corriente.

Los valores máximo y mínimo en cada una de las predicciones realizadas por las cinco instituciones consultadas reflejan que el intervalo más amplio de variación de las predicciones se produce con la estimación realizada por P3, con un valor máximo de crecimiento del PIB en 2015 del 4,9\% (La Rioja) y un valor mínimo de 1,7\% (Canarias). La mayor dispersión media se recoge en la predicción P3, seguida de P1, P4, P2, P5 y Consenso. Por tanto, las "mejores" estimaciones son las del Consenso, en primer lugar, y las realizadas por P5, en segundo 
lugar, con menores valores de RECM, PEAM y menor dispersión. Precisamente, Makridakis et al. (2009) indican que el promedio de la previsión de dos o más modelos (similar al Consenso) mejora la exactitud de la previsión y reduce la dispersión de los errores de predicción.

Tabla 4

Combinación de predicciones alternativas para el crecimiento del PIB regional en 2015 y comparativa con dato oficial de CRE-INE

\begin{tabular}{|c|c|c|c|c|c|c|c|}
\hline CC.AA. & $\begin{array}{c}\text { CRE-PIB } 2015 \\
\text { "1ª } \\
\text { Estimación" } \\
\text { marzo } 2016\end{array}$ & $\begin{array}{l}\text { P1-PIB } \\
2015\end{array}$ & $\begin{array}{l}\text { P2-PIB } \\
2015\end{array}$ & $\begin{array}{c}\text { P3-PIB } \\
2015\end{array}$ & $\begin{array}{l}\text { P4-PIB } \\
2015\end{array}$ & $\begin{array}{l}\text { P5-PIB } \\
2015\end{array}$ & $\begin{array}{c}\text { CONSENSO } \\
2015 \\
\text { Consensus } \\
\text { Forecast } \\
\Sigma P j / 5, j=1 . . .5\end{array}$ \\
\hline Andalucía & 3,2 & 3,2 & 3,0 & 2,4 & 2,9 & 3,3 & 3,0 \\
\hline Aragón & 2,7 & 3,3 & 3,0 & 3,9 & 3,1 & 3,2 & 3,3 \\
\hline Asturias & 3,1 & 2,8 & 2,6 & 2,9 & 2,3 & 2,7 & 2,7 \\
\hline Baleares & 3,2 & 4,3 & 3,9 & 4,1 & 3,1 & 2,7 & 3,6 \\
\hline Canarias & 2,8 & 3,7 & 3,3 & 1,7 & 2,2 & 3,2 & 2,8 \\
\hline Cantabria & 2,6 & 3,2 & 2,6 & 3,2 & 2,7 & 2,9 & 2,9 \\
\hline Castilla y León & 2,9 & 3,0 & 2,5 & 3,2 & 3,3 & 2,9 & 3,0 \\
\hline Castilla-La Mancha & 3,2 & 2,6 & 3,3 & 2,3 & 2,8 & 2,9 & 2,8 \\
\hline Cataluña & 3,3 & 3,6 & 3,3 & 3,1 & 3,3 & 3,4 & 3,3 \\
\hline Com. Valenciana & 3,6 & 4,3 & 3,5 & 3,5 & 3,4 & 3,5 & 3,6 \\
\hline Extremadura & 3,0 & 2,8 & 3,2 & 3,5 & 3,0 & 3,2 & 3,1 \\
\hline Galicia & 3,2 & 2,9 & 2,2 & 2,2 & 3,1 & 1,8 & 2,4 \\
\hline Com. de Madrid & 3,4 & 3,3 & 3,6 & 3,1 & 3,5 & 3,5 & 3,4 \\
\hline Murcia & 3,1 & 2,5 & 3,4 & 3,7 & 3,7 & 3,1 & 3,3 \\
\hline Navarra & 2,9 & 3,0 & 3,3 & 3,6 & 3,2 & 3,3 & 3,3 \\
\hline País Vasco & 3,1 & 3,0 & 2,6 & 3,7 & 2,8 & 2,6 & 2,9 \\
\hline La Rioja & 2,8 & 3,8 & 3,7 & 4,9 & 4,2 & 3,6 & 4,0 \\
\hline ESPAÑA & 3,2 & 3,4 & 3,2 & 3,1 & 3,2 & 3,2 & 3,2 \\
\hline valor máximo & 3,6 & 4,3 & 3,9 & 4,9 & 4,2 & 3,6 & 4,0 \\
\hline valor mínimo & 2,6 & 2,5 & 2,2 & 1,7 & 2,2 & 1,8 & 2,4 \\
\hline RECM &.. & 0,54 & 0,45 & 0,83 & 0,48 & 0,48 & 0,44 \\
\hline PEAM & .. & $14,40 \%$ & $11,58 \%$ & $22,86 \%$ & $11,43 \%$ & $11,28 \%$ & $10,31 \%$ \\
\hline
\end{tabular}

Nota: P1: AIREF (estimación realizada en noviembre 2015); P2: BBVA Research (noviembre 2015); P3: CEPREDE (diciembre 2015); P4: FUNCAS (febrero 2016); P5: HISPALINK (diciembre 2015).

Fuente: Elaboración propia a partir de datos iniciales de las instituciones señaladas.

Para valorar las diferencias de estimación es preciso resaltar que las oficinas o institutos oficiales de estadística de los diferentes países proporcionan estimaciones de crecimiento económico (variación del PIB) que posteriormente revisan. En este proceso de revisión de los datos inicialmente conocidos ("primera estimación”) y los finales (pasando por las sucesivas etapas de "avance”, "provisionales”) pueden existir diferencias cuantificadas en décimas de punto o incluso más de un punto de crecimiento por encima o por debajo del dato inicial de partida. En el proceso de revisión de predicciones de crecimiento económico es importante 
considerar la experiencia internacional. A modo de referencia, Pulido (2006) recoge un análisis de resultados referido a las predicciones de crecimiento económico de Estados Unidos realizadas por el Bureau of Economic Analysis (BEA) y concluye "la experiencia puede resumirse en que nuestra exactitud de medida es habitualmente inferior a medio punto de porcentaje en el crecimiento del PIB y que puede incluso superar al punto de porcentaje en una revisión exhaustiva realizada años más tarde”.

En este sentido, recogemos en la Tabla 5 la referencia a las mayores diferencias de estimación regional (superiores al medio punto de porcentaje) entre los datos de crecimiento del PIB de 2015 estimado por las cinco fuentes consultadas y el dato oficial publicado por la CRE-INE en marzo de 2016.

\section{Tabla 5}

Diferencias superiores al medio punto de porcentaje entre predicciones alternativas para el crecimiento del PIB regional en 2015 y comparativa con dato oficial CRE-INE

\begin{tabular}{|c|c|c|c|c|c|}
\hline P1-PIB 2015 & $\begin{array}{l}\text { P2-PIB } \\
2015\end{array}$ & P3-PIB 2015 & P4-PIB 2015 & P5-PIB 2015 & $\begin{array}{c}\text { CONSENSO } \\
2015\end{array}$ \\
\hline $\begin{array}{c}\text { Aragón } \\
\text { Baleares } \\
\text { Canarias } \\
\text { Cantabria } \\
\text { Castilla-La Mancha } \\
\text { Com. Valenciana } \\
\text { Murcia } \\
\text { La Rioja }\left(^{\star}\right)\end{array}$ & $\begin{array}{l}\text { Baleares } \\
\text { Galicia }\left(^{\star}\right) \\
\text { La Rioja }\end{array}$ & $\begin{array}{c}\text { Andalucía } \\
\text { Aragón }\left(^{\star}\right) \\
\text { Baleares } \\
\text { Canarias }\left(^{\star}\right) \\
\text { Cantabria } \\
\text { Castilla-La Mancha } \\
\text { Galicia }\left(^{\star}\right) \\
\text { Murcia } \\
\text { Navarra } \\
\text { País Vasco } \\
\text { La Rioja }\left(^{\star}\right)\end{array}$ & $\begin{array}{c}\text { Asturias } \\
\text { Canarias } \\
\text { Murcia } \\
\text { La Rioja }\left(^{*}\right)\end{array}$ & $\begin{array}{l}\text { Galicia(*) } \\
\text { La Rioja }\end{array}$ & $\begin{array}{c}\text { Aragón } \\
\text { Galicia } \\
\text { La Rioja(*) }\end{array}$ \\
\hline
\end{tabular}

Nota: P1: AIREF (estimación realizada en noviembre 2015); P2: BBVA Research (noviembre 2015); P3: CEPREDE (diciembre 2015); P4: FUNCAS (febrero 2016); P5: HISPALINK (diciembre 2015).

(*) Diferencia en la estimación superior o igual al punto de porcentaje.

Fuente: Elaboración propia a partir de datos iniciales de las instituciones señaladas.

Además de comprobarse una mayor concentración de diferencias respecto al dato oficial en el caso de la predicción realizada por P3 (once CC.AA.) y P1 (ocho CC.AA.) y menores errores cometidos por P5 y Consenso, puede apreciarse una coincidencia en todas las estimaciones alternativas de crecimiento del PIB regional en 2015 respecto a "fallar" en la predicción realizada en el caso de La Rioja. Esta comunidad autónoma lideró el crecimiento económico regional en 2014 con una tasa de variación del PIB del 2,0\% frente al 1,4\% de España, contabilizando un diferencial positivo de crecimiento de seis décimas de punto. Si se hubiera mantenido este diferencial en 2015, habría supuesto un crecimiento de La Rioja del orden del 3,8\% en tasa de variación del PIB (España creció un 3,2\%). Todas las predicciones alternativas apuntan un crecimiento del PIB de La Rioja en 2015 superior al 3,6\%, mientras que la realidad, según el dato oficial de CRE- 
INE, ha sido un crecimiento del 2,8\%, cuatro décimas inferior al promedio nacional $(3,2 \%)$.

Se aprecia mejor la adecuación de los datos estimados a los datos oficiales publicados en el gráfico correspondiente al diagrama de predicción-realización (Figura 4) donde se compara la previsión de Consenso de las cinco fuentes alternativas consultadas y el dato oficial de crecimiento del PIB regional en 2015. El PEAM del consenso implica que el pronóstico falla en un 10,31\%. Los casos en los que convendría revisar los procesos de predicción individual se corresponden con las comunidades autónomas de La Rioja, Galicia y Aragón donde las diferencias superan el medio punto de porcentaje. Estas tres comunidades autónomas comparten la importancia que el sector industrial tiene en su estructura productiva y que, posiblemente, sea el causante del desajuste en la predicción combinada. Aunque también es cierto que habría que considerar los matices que aportan los subsectores industriales (intermedios, consumo, equipo) en cada caso y que pueden no haber sido considerados expresamente en el proceso de predicción global.

Figura 4

Diagrama Predicción-Realización del Consenso de predicciones alternativas de crecimiento del PIB regional en 2015 y comparativa con dato oficial de CRE-INE

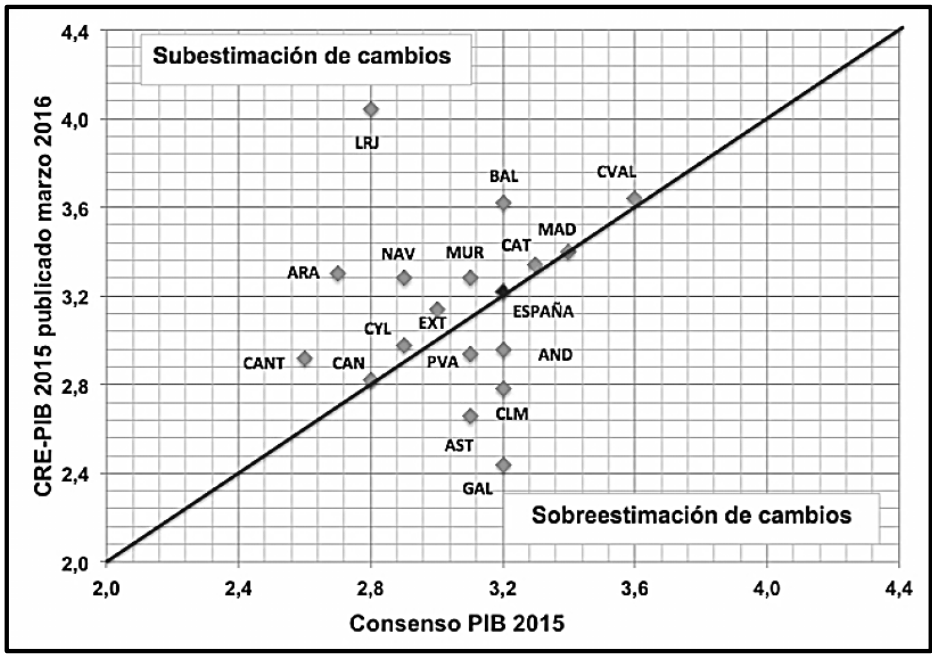

Fuente: Elaboración propia a partir de datos iniciales de CRE-INE y de las instituciones señaladas.

Finalmente, proporcionando respuesta al interrogante sobre la predicción más adecuada, en términos del error de estimación cometido parece deducirse más conveniente la propuesta de modelización procedente de un enfoque metodológico tipo bottom-up y con detalle sectorial (P5) que obtiene el menor de los 
PEAM presentados individualmente (11,28\%). Una posibilidad adicional sería ponderar de forma distinta la participación de cada estimación alternativa (desde P1 hasta P5), en lugar de otorgar el mismo peso lineal a cada una (1/5). Se han realizado comprobaciones combinando las distintas estimaciones, sin considerar conjuntamente a las cinco instituciones, hasta alcanzar una combinación donde el PEAM fuese menor que el obtenido en el consensus forecast. Ha sido posible combinando sólo P2 y P5, obteniendo un PEAM de un 10,00\%. No obstante, parece prudente no descartar aún ninguna de las otras estimaciones alternativas, procediendo a la combinación de todas las predicciones, pues, en conjunto, alcanzan un error de pronóstico del 10,31\% que puede calificarse como asumible (el óptimo sería un error del $0 \%$ ), especialmente si se compara con el propio error de estimación cometido por el INE en la publicación del dato referido al año 2014 (PEAM 43,5\%, Tabla 3).

\section{CONCLUSIONES}

En economía, los datos (series temporales) posibilitan la elaboración de indicadores compuestos y modelos (información) que ayudan en el proceso de interpretación previo a la generación del conocimiento. La sucesión entre los tres conceptos definidos es de forma jerárquica, donde cada nivel es construido sobre la base del anterior, dotando al conocimiento de la máxima importancia en todo el proceso. No obstante, más información no siempre genera una mejor comprensión del conocimiento económico.

En el ámbito concreto de la predicción económica, un deseo inherente a los analistas económicos es poder predecir la evolución de una economía, su ritmo de crecimiento, para anticiparse a la toma de decisiones o a la aplicación concreta de políticas económicas. Las predicciones de crecimiento del PIB como instrumento para extrapolar el comportamiento futuro de una economía contribuyen a la generación de conocimiento económico. La tasa de variación del PIB, como medida del crecimiento económico de un país o región, precisa ser interpretada correctamente para extraer conclusiones relevantes en la toma de decisiones económicas, en la generación y difusión de conocimiento. La publicación y difusión de predicciones de crecimiento económico pueden repercutir o incluso influir en las decisiones o comportamiento de los agentes, generan conocimiento y por ello es importante valorar la fiabilidad de la predicción. Las personas y las organizaciones comúnmente toman decisiones combinando la información de múltiples entradas, y en ese proceso la predicción es útil (Graefe, 2015).

El proceso de revisión de los principales aspectos a considerar en el análisis de predicciones del PIB regional en España, se ha realizado con la finalidad de contribuir a esclarecer el interés por la combinación de predicciones alternativas o la apuesta por una predicción en particular por su mayor fiabilidad o preci- 
sión. En el caso particular del PIB regional en España se han extraído conclusiones relevantes respecto a la eficacia predictiva en el año 2015, atendiendo al análisis de la combinación de predicciones. Al tratarse de un año ya finalizado, la dispersión entre la predicción nacional y la que se corresponde con la agregación de predicciones regionales siempre se reduce a medida que nos acercamos al instante para el que se predice y con mayor consenso sobre lo que finalmente aconteció según los datos oficiales. Como también se expone en OCDE (2014), las diferentes predicciones de crecimiento presentan una mayor coincidencia al aproximarse al cierre del año corriente.

Podemos concluir en la conveniencia de recurrir a las predicciones para poder anticipar la evolución futura de la situación económica de las comunidades autónomas en España. Sin duda, en este proceso de análisis el dato inicial se va conformando en información y ésta en conocimiento económico.

En el análisis realizado, las "mejores” estimaciones son las del Consenso o combinación de predicciones, con valores relativos inferiores de RECM, PEAM y menor dispersión. Precisamente, en línea con lo argumentado por Makridakis et al. (2009) que indican que el promedio de la previsión de dos o más modelos (similar al Consenso) mejora la exactitud de la previsión y reduce la dispersión de los errores de predicción.

Las estimaciones realizadas por una institución (P5) cuya propuesta de modelización procede de un enfoque metodológico tipo bottom-up se sitúan en segunda posición como predicción más adecuada, frente al resto que optan por enfoques tipo top-down, en términos del error de estimación cometido, pues contabiliza los menores valores de RECM y de PEAM de los presentados individualmente.

Sería deseable un estudio en un periodo más amplio, pero actualmente está limitado por la información disponible de las fuentes predictoras utilizadas, que posibilitase aplicar los planteamientos del trabajo realizado por la OCDE (2014) donde se analiza la eficacia de previsiones de la OCDE durante el período 200712. En tal periodo se pueden constatar las diferencias entre países en los errores de predicción de crecimiento, con sesgo de sobreestimación en general, y los cambios en los modelos de predicción y procedimientos que han sido impulsados por la experiencia de la crisis.

El proceso de predicción regional no es un camino cerrado, aún quedan opciones por explorar y desarrollar, no es una finalidad en sí mismo, sino un medio para elaborar información (predicciones regionales) que genere conocimiento sobre las perspectivas de evolución regional que interese a los analistas regionales y a la sociedad en general. Es importante también considerar que los procedimientos técnicos utilizados para la predicción económica siempre deberían ser lo bastante simples como para ser entendidos por cualquier usuario de las predicciones (Green y Armstrong, 2015). 
La consulta de predicciones económicas es una labor que sirve para valorar el grado de confianza de las predicciones y debe ser una ayuda para la correcta interpretación del marco económico que subyace en las políticas presupuestarias regionales (Directiva 2011/85/UE del Consejo de 8 de noviembre de 2011 sobre los requisitos aplicables a los marcos presupuestarios de los Estados miembros). En cualquier caso, el proceso siempre merecerá la pena: "Prefiero estar aproximadamente en lo cierto que exactamente equivocado” (Keynes, 1973).

\section{REFERENCIAS BIBLIOGRÁFICAS}

AIREF (2015). Quarterly regional GDP flash estimates for the spanish economy (Metcap model), working paper DT/2015/3, mayo.

ALCAIDE, J. (1996). "Contabilidad regional de las autonomías españolas. Un modelo simplificado". Papeles de Economía Española, nº 67, FUNCAS, Madrid, pp. 2-45.

ALCAIDE, J. y ALCAIDE, P. (2000). "El crecimiento económico de las autonomías españolas en 1999". Cuadernos de Información Comercial Española, Los ejes de la economía española, n 155 , pp. 1-49, marzo-abril.

ALVIRA, F. y GARCÍA, J. (2000). "La evolución de la economía en las comunidades autónomas: percepción social frente a indicadores objetivos". Cuadernos de Información Económica, nº 155, marzo-abril, pp. 98-106.

ARRAZOLA, M.; HEVIA, J.; MAULEÓN, I. y SÁNCHEZ, R. (2011). "Estimación del volumen de economía sumergida en España". Cuadernos de Información Económica, $\mathrm{n}^{\circ}$ 220, pp. 81-87.

BAKER, S.R.; BLOOM, N. y DAVIS, S.J. (2015). Measuring Economic Policy Uncertainty. Centre for economic performance CEP Discussion Paper $\mathrm{n}^{0} 1379$, october.

BOLTON, R. (1991). "Regional Econometric Models". En BODKIN R.; KLEIN L.R. y MARWAH K. (ed.): A History of Macroeconometric Model-Building, pp. 451-481, EDWARD ELGAR P.L.

BURNS, A.F. y MITCHELL, W.C. (1946). Measuring Business Cycles. NBER, Studies in Business Cycles, $n^{\circ} 2$, New York.

CALLEALTA, F.J. y LÓPEZ, A.M. (2001). "Proceso de estimación de predicciones regionales: el modelo de congruencia de Hispalink". En CABRER, B. (coord.): Análisis Regional: El Proyecto Hispalink, (pp. 31-41). Madrid: Mundi Prensa.

CAMACHO, M. y DOMÉNECH, R. (2010). MICA-BBVA: a factor model of economic and financial indicators for short-term GDP forecasting. BBVA Economic Research Working papers, $\mathrm{n}^{\circ} 1021$.

DAVENPORT, T.H. y PRUSAK, L. (1998). Working Knowledge: How Organizations Manage What They Know, Harvard Business School Press.

DIEBOLD, F.X. (1997). The past, present and the future of macroeconomic forecasting. Working paper 6290, NBER working paper series, Cambrigde Massachusetts.

DIEBOLD, F.X. y PAULY, P. (1990). "The Use of Prior Information in Forecast Combination". International Journal of Forecasting, $\mathrm{n}^{\circ}$ 6, pp. 503-508. 
ENGLE, R.F.; GRANGER, C.W.J. y KRAFT, D.F. (1984). "Combining Competing Forecasts of Inflation Using a Bivariate ARCH Model". Journal of Economic Dynamics and Control $\mathrm{n}^{\circ}$ 8, pp. 151-165.

ESPASA, A. (1980). La predicción económica. Estudios Económicos, nº 18, Servicio de Estudios Banco de España.

FRIEDMAN, M. (1953). Essays in Positive Economics, The University of Chicago Prest.

GRAEFE, A. (2015). "Improving forecast using equally weighted predictors". Journal of Business Research, n 68, pp. 1792-1799.

GREEN, K.C. y ARMSTRONG, J.S. (2015). "Simple versus complex forecasting: the evidence". Journal of Business Research, n 68, pp. 1678-1685.

GUIDETTI, G. y GYOMAI, E. (2012). OECD System of composite leading indicators, OECD.

HAYEK, F. (1945). "The Use of Knowledge in Society". The American Economic Review, vol. XXXV, no 4, september, pp. 519-530.

HONG, Y. (2006). Understanding modern econometrics, WISE working paper series WISEWP0611. Wang Yanan Institute for Studies in Economics, Xiamen University, China.

HUANG, H., LEE, T. (2007). To Combine Forecasts or to Combine Information?. Department of Economics University of California, Riverside, january.

KANTOROVICH, L.V. (1975). "Las Matemáticas en Economía: logros, dificultades y perspectivas" discurso de aceptación del premio Nobel de Economía. En Lindbeck, A. (ed.) Nobel Lectures, Economics Sciences 1969-1980, World Scientific Publishing Co., Singapore, 1992.

KEYNES, J. M. (1973). Collected Writings de John Maynard Keynes Macmillan. London, Tomo VII Treatise on Probability.

KOSE, M.A. Y TERRONES M.E. (2015). Collapse and Revival: Understanding Global Recessions and Recoveries. Washington D. C.: International Monetary Fund.

KLEIN, L.R. (1969). "The Specification of Regional Econometric Models". Papers in Regional Science, vol. 23, 1, pp. 105-116.

LAHIRI, K. y MOORE, G. H. (1991). Leading economic indicators: new approaches and forecasting records. New York: Cambridge University Press.

LÓPEZ, A.M. (2015). "Modelización econométrica regional en España: una revisión aplicada del enfoque unirregional y multirregional". Estudios de Economía Aplicada, vol. 33(2), mayo, pp. 399-420.

LOUNGANI, P. (2001). "How accurate are private sector forecasts? Cross-country evidence from consensus forecasts of output growth". International Journal of Forecasting, vol. 17, issue 3, july-september, pp. 419-432.

LUCAS, R. (1977). "Understanding Business Cycles". Carnegie-Rochester Conference Series on Public Policy, vol. 5, pp. 7-29.

MAKRIDAKIS, S.; HOGARTH, R. y GABA, A. (2009). "Forecasting and uncertainty in the economic and business world". International Journal of Forecasting, vol. 25, issue 4, october-december, pp. 794-812.

MARSHALL, A. (1890). Principles of Economic. London: MacMillan. (versión española de la octava edición inglesa de 1920, Marshall, A. (1948): Principios de Economía. Madrid: Aguilar).

MERIGUETE, D. y PÉREZ, J. (2014). Indicadores de alta frecuencia para el crecimiento 
regional y su obtención a través de desagregación temporal con restricción, Documento de trabajo Instituto L. R. Klein-Centro Stone/CEPREDE.

OCDE (1999). OECD Composite Leading Indicators: a tool for short-term analysis, OECD Economics Department.

OCDE (2010). OECD Cyclical Analysis and Composite Indicators System, User Guide, OECD Economics Department.

OCDE (2014). OECD Forecasts during and after the financial crisis: a post mortem, OECD Economics Department, Policy Note $n^{\circ} 23$, febrero.

PÉREZ, R.; LÓPEZ, A.J.; MORENO, B.; RODRIGUEZ, S.; CALLEALTA, F.J.; LÓPEZ, A.M. y BUENDÍA, J.D. (2009). "Predicción económica regional: experiencias de la red Hispalink". Información Comercial Española, Revista de Economía, n 848, mayo-junio, pp. 141-158.

PHILLIPS, P.C. (2004). Laws and limits of econometrics. Cowles Foundation paper $\mathrm{n}^{\circ} 1081$. Cowles Foundation for research in economics, Yale University, New Haven, Connecticut.

PIFFANO, H. L. P. (2012). Las Crisis y los Modelos Económicos: Diagnósticos, Predicciones y las Políticas Económicas. Documento de Trabajo no 93 octubre, Departamento de Economía, Facultad de Ciencias Económicas, Universidad Nacional de La Plata.

PONS, J. (1999). Previsiones de crecimiento de la economía española: una valoración. ICE Tribuna de Economía, n 780, septiembre, pp. 143-153.

PULIDO, A. (2006). "La aventura de la predicción económica y empresarial". En Pulido, A. (coord.): Guía para usuarios de predicciones económicas (pp. 9-30). Madrid: Ecobook.

PULIDO, A. (2015). "La herencia de Klein (1920-2013): una visión de futuro". Estudios de Economía Aplicada, vol. 33(2), mayo, pp. 359-384.

PULIDO, A. y LÓPEZ, A.M. (1999). Predicción y simulación aplicada a la economía y gestión de empresas. Madrid: Ed. Pirámide.

RICKMAN, D.S. (2014). "Macroeconomía moderna y modelación económica regional". Paradigma económico, año 6, n¹, pp. 5-30.

SOLOW, R. (1987). "We'd better watch out". New York Times Book Review, july 12, página 36.

STIGLITZ, J.E.; SEN, A. y FITOUSSI, J.P. (2008). Informe de la Comisión sobre la Medición del Desarrollo Económico y del Progreso Social, www.stiglitz-sen-fitoussi.fr.

THEIL, H. (1961). Economic Forecast and Policy. North Holland Publishing.

THEIL, H. (1966). Applied Economic Forecasting. North Holland Publishing.

VICÉNS, J. y MEDINA, E. (2014). Evaluación de predicciones macroeconómicas. enero, $n^{\circ}$ 24, Documento de Trabajo Instituto L.R.Klein-Centro Gauss, Universidad Autónoma de Madrid. 
